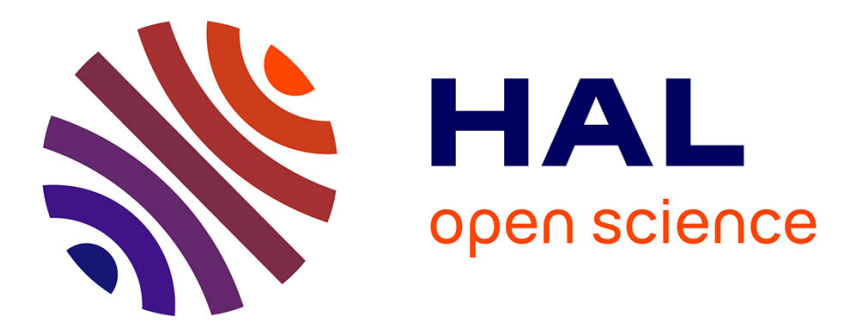

\title{
Optimal magnetic sensor location for spherical harmonics identification applied to radiated electrical devices
}

Laure-Line Rouve, Louis Schmerber, Olivier Chadebec, Albert Foggia

\section{To cite this version:}

Laure-Line Rouve, Louis Schmerber, Olivier Chadebec, Albert Foggia. Optimal magnetic sensor location for spherical harmonics identification applied to radiated electrical devices. IEEE Transactions on Magnetics, 2006, 42 (4), pp.1167-1170. 10.1109/TMAG.2006.872016 hal-00164517

\section{HAL Id: hal-00164517 https://hal.science/hal-00164517}

Submitted on 27 Jun 2008

HAL is a multi-disciplinary open access archive for the deposit and dissemination of scientific research documents, whether they are published or not. The documents may come from teaching and research institutions in France or abroad, or from public or private research centers.
L'archive ouverte pluridisciplinaire $\mathbf{H A L}$, est destinée au dépôt et à la diffusion de documents scientifiques de niveau recherche, publiés ou non, émanant des établissements d'enseignement et de recherche français ou étrangers, des laboratoires publics ou privés. 


\title{
Optimal Magnetic Sensor Location for Spherical Harmonic Identification Applied to Radiated Electrical Devices
}

\author{
Laure-Line Rouve ${ }^{1}$, Louis Schmerber ${ }^{1}$, Olivier Chadebec ${ }^{2}$, and Albert Foggia ${ }^{2}$ \\ ${ }^{1}$ Magnetism Laboratory of Grenoble Franc (LMN), 38402 St Martin d'Hères, France \\ ${ }^{2}$ Electrical Engineering Laboratory of Grenoble France (LEG), 38402 St Martin d'Hères, France
}

\begin{abstract}
This paper deals with the use of spherical harmonic representation to identify the magnetic multipolar sources associated to a given item, by measuring the near field around it. If the model presents many advantages, its use in an inverse problem approach requires special caution to critical points like the choice of sensor number and location. A method is presented that makes it possible, from a high number of information, to reduce the sensor number to a minimum and to optimize their location with respect to robustness. The robustness issue is solved by using an original method based on a genetic algorithm. A simple but didactic example illustrates the difficulties that can be met and our strategy to overcome them.
\end{abstract}

Index Terms-Electric machines, harmonic analysis, inverse problems, magnetic field measurment.

\section{INTRODUCTION}

$\mathbf{F}$ OR many applications, it is interesting to determine a model for the magnetic sources that compose an electrical device. Thanks to this model, it is then possible to know the induction all around the structure, in particular far away where sensors may not be placed. The applications can be various: electromagnetic compatibility, magnetic shielding, military naval discretion, etc.

If the geometry and the magnetic properties of the device are well known, a direct approach (based on finite-element [1] or boundary-integral methods) can be adopted. We would like now to focus on items for which nothing is known about its sources. It is clearly the worst case to deal with because no a priori information can be used. As a consequence, an inverse problem approach must be applied. To do so, a representative model of the sources must be chosen. By using information (near field measurement) the model is identified. It can be noticed that the identification can also be applied after a direct computation to obtain another model for the sources, that can be easier to handle than the initial one.

A model based on spherical harmonics [2] presents specific advantages that make it a good candidate for inversion. This model is quite well known and has already been used in the past [3]. Nevertheless, not a lot of answers have been given to this general problem: the equivalent sources of an object must be identified, nothing is known about it; where sensors must be located? How many are required to well solve the problem? Is there an optimum number for that? Is the identification robust with respect to noise sensors?

If a measurement system makes it possible to have a high number of information at a distance from the device, the multipolar sources can be identified [4]: the problem is over sized. What happens if, for instance, only to check the variation of

Digital Object Identifier 10.1109/TMAG.2006.872016 the device magnetic state, the same identification should be achieved with only a few sensors?

The paper deals with a method that studies how to well identify the multipolar sources in a over sized case and then with a minimum number of sensors. One specificity for the sensors is that their location is defined by their robustness with respect to (sensor) noise. A method based on genetic algorithms which minimize the matrix conditioning of the inverse problem finds the best setting for sensors. In order to also increase robustness, attention is paid to the choice of the center for the decomposition. A simple but educational example illustrates the general approach.

\section{SPHERICAL HARMONIC IDENTIFICATION}

\section{A. Spherical Harmonic Model}

The solving of Laplace's equation in spherical coordinates leads to the following expression for magnetic induction $\mathbf{B}$ created by an electrical device included in a volume $(V)$

$$
\mathbf{B}(r, \theta, \varphi)=-\frac{\mu_{0}}{4 \pi} \sum_{n=1}^{\infty} \sum_{m=-n}^{n} a_{n, m} \cdot \vec{\nabla}\left(\frac{1}{r^{n+1}} \cdot Y_{n}^{m}(\theta, \varphi)\right)
$$

$$
\begin{array}{ll}
\text { where } & \\
\mu 0=4 \pi 10^{-7} & \text { (unit of IS); } \\
n & \text { an order of decomposition; } \\
m & \text { associated suborder; } \\
\mathbf{Y}_{\mathbf{n}}^{\mathrm{m}} & \text { spherical harmonic functions; } \\
(r, \theta, \phi) & \text { spherical coordinate for } P \text { a point outside }(V) \\
& \text { where the induction is expressed; } \\
a_{n, m} & \text { are called spherical harmonic coefficients. }
\end{array}
$$

\section{B. Spherical Harmonic Model Properties}

The $a_{n, m}$ coefficients are intrinsic to the source and to the choice of the decomposition center. Therefore, they do not depend on measurements but only on the source. Each of them are 
linked to a multipolar representation [5] with specific periodicities. Order 1 corresponds to a dipole, order 2 to a quadripole, etc.

Moreover, for a given order $n, a_{n, m}$ coefficients are associated to a $1 / r^{n+2}$ decreasing law, where $r$ is the distance from the measurement point to the center of the decomposition. As a consequence, if we are far enough from the sources, the induction contribution of high orders becomes negligible. Thus, the infinite sum can be truncated to an order $N_{\max }$ while ensuring a good representation for the field. It is important to specify what negligible contribution means. This means that the induction contribution becomes lower than a given limit. This limit can be sensor resolution during experiment or computer precision regarding computation results.

Finally, the magnetic field in (1) linearly depends on the $a_{n m}$ coefficients. It is possible to use this property to build a matrix system that will be studied for inversion.

\section{Inverse Problem}

The aim is to determine the $a_{n, m}$ coefficients of the model from information, i.e., the magnetic field around the studied device. This problem is a problem of identification (or inverse problem).

If the decomposition is limited at the order $N_{\max }$ and if $K$ is the sensor number (point where information is collected), a linear system which links the coefficients to the information points is built

$$
\underline{\mathbf{B}} m e s=\underline{\underline{\mathbf{G}}} \cdot \underline{\mathbf{A}}
$$

where $\mathbf{B}$ mes is a $3 K$-dimension vector of information (induction components on all the sensors), $\mathbf{A}$ is a $\left(N_{\max }^{2}+2 N_{\max }\right)$-dimension vector of the different $a_{n, m}$ coefficients, and $\mathbf{G}$ is a $\left(3 K, N_{\max }^{2}+2 N_{\max }\right)$-dimension matrix of spherical harmonic influences on sensors.

As spherical harmonics constitute an orthogonal basis for magnetic induction, this point ensures a quite well-posed system.

The system (2) has to be solved to get all the $a_{n, m}$ coefficients. If the number of information given by the magnetic sensors ( $3 * K$ induction components) is smaller than the number of the $a_{n, m}$ coefficients ( $N_{\max }^{2}+2 N_{\max }$ for a $N_{\max }$-order source) the system is rank-deficient and resolution is a physical nonsense. In this work, the two other scenarios are treated: in the first case, the problem is oversized (the information number is higher than the number of unknowns); in the second case, as we want to be sure to well solve the problem with the minimum sensor number, we choose to solve a square problem (the number of unknown coefficients is equal to the number of information). The system is solved by a singular value decomposition (pseudoinversion).

To decrease the matrix condition number, the unknown coefficients are also normalized.

\section{Conditions for a Well-Posed Problem}

As it has been seen before, for a given signal-to-noise ratio and a given radius $R$, the order $N_{\max }$ is enough to correctly

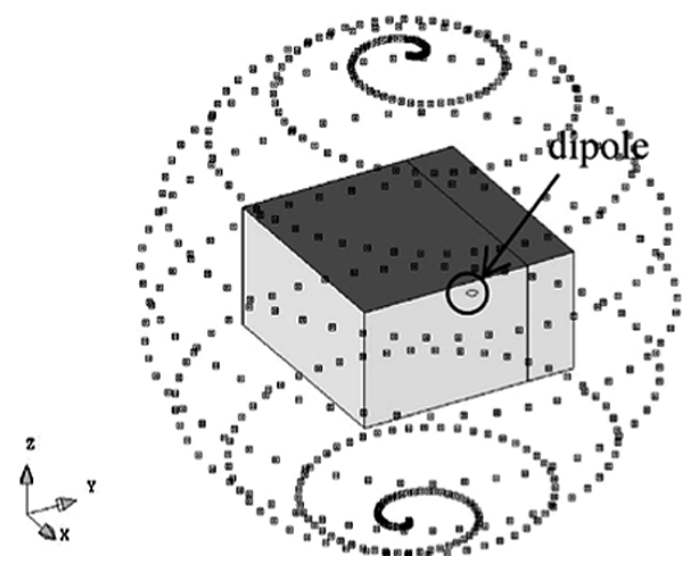

Fig. 1. Geometry of the studied device represented by a shifted dipole $(M=$ $63 \mathrm{Am}^{2}$, located at $[1.5 ; 0.5 ; 0.5]$ ) included inside a shield (square base of side $a=4.5 \mathrm{~m}$ and height $h=2.5 \mathrm{~m}$ ).

compute induction far from the sphere $(S)$ defined by its center $O$ (center for the harmonic decomposition), and radius $R$. If $N_{\max }$ is defined, then the number of unknowns is deduced $\left(N_{\max }^{2}+2 N_{\max }\right)$, and as a consequence, the minimum information number is also defined to well inverse the problem.

Regarding the available sensor number, the main difficulty is to determine $N_{\max }$ associated to the chosen center $O$, the radius $R$, and the studied device.

As we will see later, for some cases, the maximum order $N_{\max }$ may decrease with the choice for the center of harmonic decomposition. Thus, so do the sensor number.

Indeed, the further (the higher $R$ ), the less sensor number is required but, unfortunatel,y the lower signal-to-noise ratio is also received.

Once these three points defined $\left(N_{\max }, R, O\right)$, the last point to determine is the location of sensors on the identification sphere $(S)$. This location must be robust enough to preserve the solving accuracy from the noise inherent to information (computation or sensor noises).

\section{THE STUDIED DEVICE}

Our approach is illustrated through a didactic example.

The studied device is represented by a parallelepiped (square base of side a and height $h$ ) made of magnetic material (relative permeability of 1000), inside which a shifted dipole is included (Fig. 1).

This device is modeled thanks to a finite-element software. Its mesh is fine enough not to have a high numerical noise on the external computed induction. The interest of this kind of model is that induction information is easily for any number of points.

We define $\left(S_{0}\right)$ the minimum sphere of center $O$ (center of the shielded parallelepiped) and radius $r_{0}\left(r_{0}=3.5 \mathrm{~m}\right)$ that includes the whole device.

We also define a spiral curve $(\Gamma)$ located on a sphere centered on $O$ and of various radii $r_{n}$ [for example $r_{1}=4 \mathrm{~m}, r_{2}=5 \mathrm{~m}$ (see Fig. 1) and $r_{3}=7 \mathrm{~m}$ ]. This curve makes it possible to verify if the result of identification is correct or not by comparing it to finite element computation. 

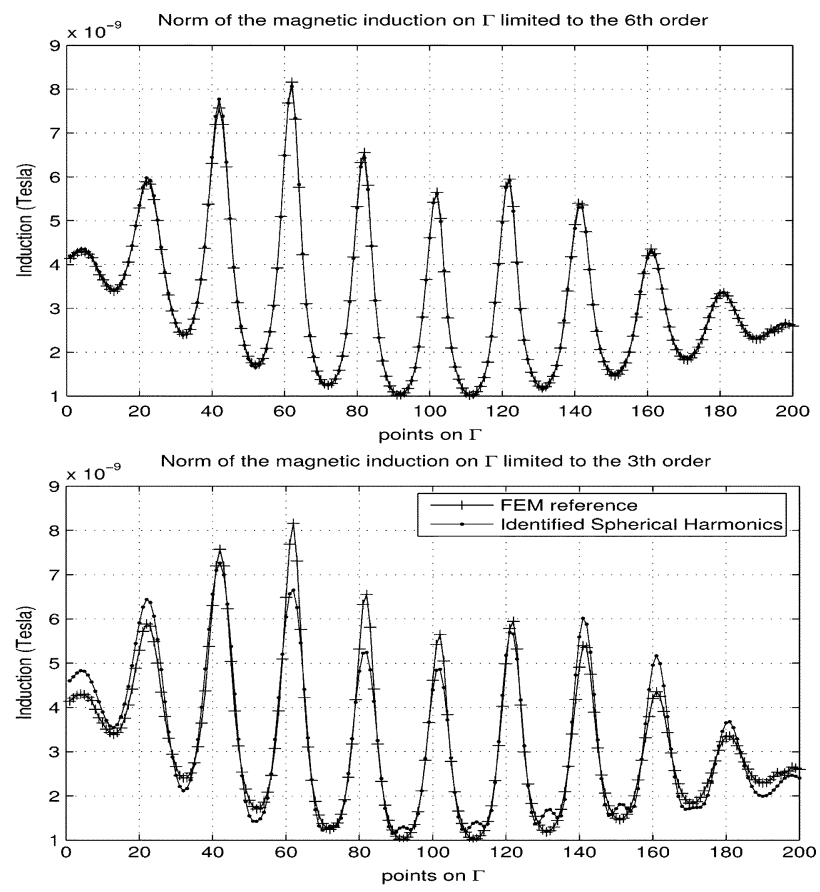

Fig. 2. Comparison of the induction modulus computed on $(\Gamma)$ spiral curve $(r 1=4 \mathrm{~m})$ with the finite-element model and the multipolar model when $N_{\max }=6$ (up) or $N_{\max }=3$ (down).

\section{A DiDACTICAL EXAMPLE}

\section{A. $N_{\max }$ Determination in the Over Sized Case}

Identification is firstly made by using a high number of information: 540 induction component values (equivalent to 180 triaxial sensors) given by the finite element model on a cube $(\Sigma)$ tangent to $\left(S_{0}\right)$. As the maximum order of the source is unknown, identification is achieved by assuming a high maximum order (22), and then for lower orders (until 10). The $a_{n, m}$ coefficients that are obtained present a great variance for high orders. As the $a_{n, m}$ coefficients are intrinsic to the source, the computed coefficients that exhibit a high variance are correlated to numerical noise and the ones that are not affected can be linked to the real device sources. At the end, the device has a 6-maximum-order $N_{\max }$.

The checking on the $(\Gamma)$ spiral curve $(r 1=4 \mathrm{~m})$ confirms this result by comparing the multipolar model with $N_{\max }=6$ (good agreement) and $N_{\max }=3$ (high discrepancy) (Fig. 2). Let us note that as the finite-element results have very low noise and as a lot of information is used, even if the coefficients have been identified on further points, they can give good accuracy really close to the device.

\section{B. Influence of the Choice for the Decomposition Center}

If the center $O$ is used for the model, the 6-maximum-order $N_{\text {max }}$ involves that 16 triaxial sensors are required for a confident identification on sphere $\left(S_{0}\right)$.

The choice of center $O$ for decomposition comes naturally with respect to the external shape of the studied device. Nevertheless, in our example, the equivalent magnetic center clearly defers from the geometrical center $O$ that has firstly been chosen for identification. This may be the case for a lot of items that

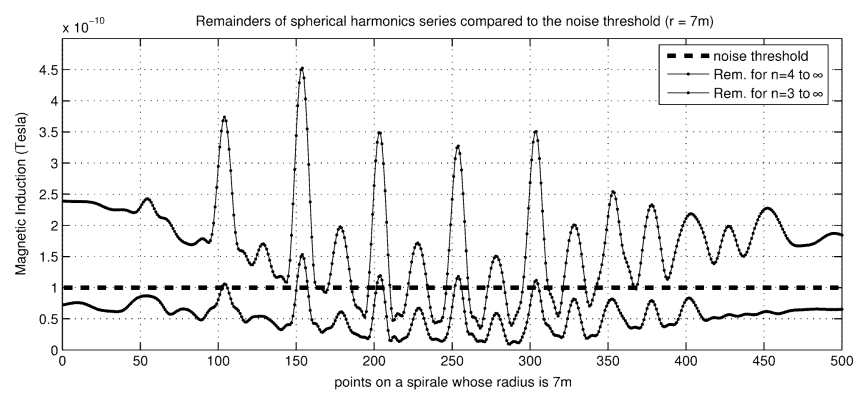

Fig. 3. Induction contribution for high orders on $(\Gamma)$ spiral curve $\left(r_{3}=7 \mathrm{~m}\right)$.

present an external casing inside which magnetic sources are included. For this configuration, the choice of another center makes it possible to have a lower maximum order $N_{\max }$ for the multipolar development.

An optimization algorithm is used to find the best center for which the norm of 5 and 6 order coefficients are minimized and the lower order ones maximized. This algorithm computes information on new minimum spheres that include the device, that are centered on point $O^{\prime}$ and that has a radius $R^{\prime}$. Information is obtained by using the initial model centered on $O$. New order $N_{\max }^{\prime}$ is determined as in the previous section.

After optimization, a new center is found (quite close to the dipole source) that corresponds to a new maximum order equal to 3 and a minimum radius $R^{\prime}$ of $5 \mathrm{~m}$ [the associated sphere is noted $\left(S^{\prime}\right)$ ] As a consequence, a confident solving can now be made with only 5 triaxial sensors located on $\left(S^{\prime}\right)$, instead of 16 located on $(S)$.

\section{Influence of the Choice for the Radius $R$}

Until now, identification has been made as close as possible to the device in order to get the higher signal-to-noise ratio.

Another way to work with a lower maximum order, while keeping the initial center $O$, is to put sensors far enough from the device. Thus, for a given radius $R^{\prime \prime}$, the total induction contribution due to higher orders becomes naturally negligible with respect to sensor noise level.

The noise level is $0.1 \mathrm{nT}$ for example. Then, the contribution for orders from 4 to $\infty$ is lower than the noise at $7 \mathrm{~m}$ far from $O$ (Fig. 3). As a consequence, $N_{\max }$ is equal to 3 . Nevertheless, the signal-to-noise ratio becomes also weak. This means that identification will be affected by the sensor noise whereas it is not the case for identification on $\left(S^{\prime}\right)$ whose center is $O^{\prime}$ and radius is $5 \mathrm{~m}$.

\section{Location of Sensors: Robustness With Respect to Noise}

To work with minimum sensor number (i.e., lower $N_{\max }$, equal to 3) while measuring the higher induction signal, we want now to put the five required sensors on $\left(S^{\prime}\right)$ and to be sure that the identification will be robust with respect to sensor noise.

As noticed previously, the system to inverse is a quite well-posed problem. But if equations are too parallel (closed to the contradiction), a small noise on measurements can lead to a divergent solution. A good indicator for the stability of an inversion process is given by the condition number of the direct matrix $\mathbf{G}$. 


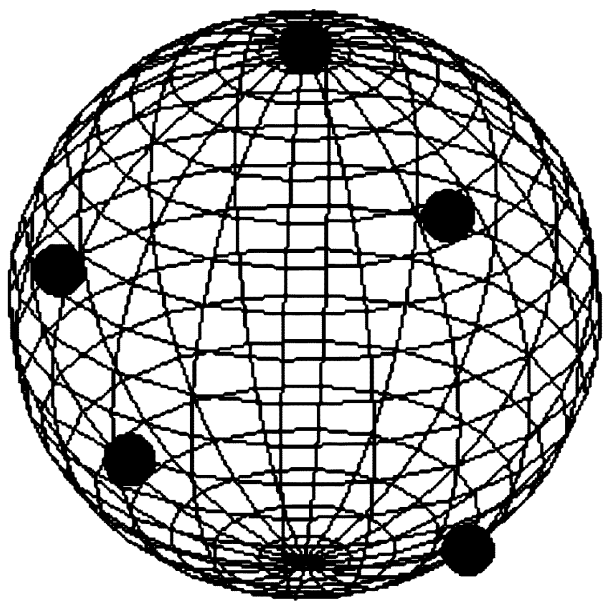

Fig. 4. Optimal sensor configuration obtained by the genetic algorithm.

A genetic algorithm is used to find the optimum location for the five sensors by minimizing the condition number of the direct matrix $\mathbf{G}$.

Sensor position are forced on the sphere $\left(S^{\prime}\right)$ (ten parameters). The objective function is the condition number. We choose a population of 40 individuals and a number of 1000 generations. An optimum location is found for the five sensors (Fig. 4).

One advantage for the solution is that this procedure is totally independent from the equivalent multipolar sources. It is only linked to spherical harmonic functions, to the number of sensors and to their position.

Then a new identification is made to test if this location is really robust with respect to sensor noise. Induction is computed on sensors. The initial multipolar model [that was identified on $(S$ ] and whose maximum order was 6) is used. It gives the same values as finite element model. Previous noise is then added on each sensor components before inversing the problem and finding the $a_{n, m}$ coefficients.

To validate the identification, the new model is used to compute induction modulus on the centered spiral curve $(\Gamma)$ whose radius $r_{3}$ is $7 \mathrm{~m}$. Fig. 5 shows a good agreement with finite element model. In spite of the noise, the inversion is correctly made.

\section{CONCLUSION}

In this paper, a method is presented to identify an equivalent multipolar source model (based on spherical harmonic functions) by near field measurements.

For a given source, the main difficulty is to well estimate its associated maximum order that allows to limit the infinite development to a finite one. In a first step, this maximum order is determined by using a lot of information around the device. This information consists of magnetic induction triaxial components.

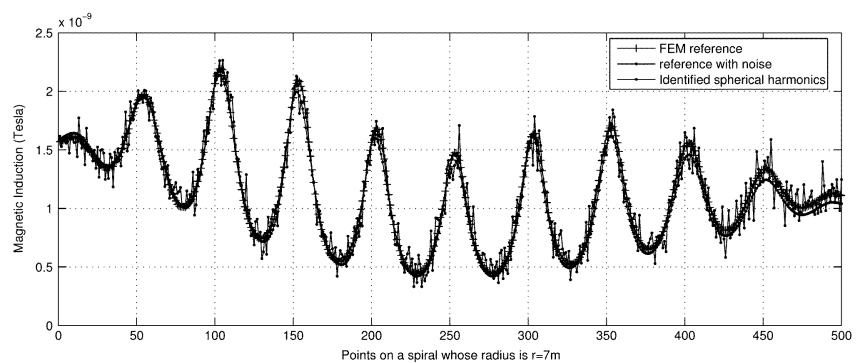

Fig. 5. Test of the multipolar model identification (based on five sensors located on the shifted sphere $\left(S^{\prime}\right)$ at a position optimized with respect to noise sensor) on the centered spiral curve $(\Gamma)$ whose radius $r_{3}$ is $7 \mathrm{~m}$.

It can be obtained from a direct modeling (based on finite element or boundary and integral methods) or from a measurement system. The maximum order is determined by studying the variance of identified model coefficients.

Then, a method is given to choose the best center for spherical development. A good choice may make it possible to decrease the maximum order. It must be lower as possible because it conditions the minimum sensor number that ensures to get a good identification. The distance where sensors are located also strongly influences the value for this maximum order but special attention must be paid to signal-to-noise ratio.

Once maximum order, decomposition center and sensor distance are clearly determined, it is then possible to only use a few sensors around the device while making an exact identification. For inversion, the sensor position is also crucial: an optimization method is presented that makes it possible to have a robust inversion with respect to the noise.

Finally, a robust procedure has been defined to determine the magnetic state of a device from a minimum sensor number around it.

\section{ACKNOWLEDGMENT}

This work was supported in part by the French Navy.

\section{REFERENCES}

[1] B. Froidurot, "Magnetic discretion of naval propulsion machines," $J$. Magn. Magn. Mater., vol. 242-245, pp. 1190-1194, Apr. 2002.

[2] E. W. Hobson, The Theory of Spherical and Ellipsoidal Harmonics. New York: Chelsea, 1955.

[3] R. Scorreti et al., "Estimation of an equivalent multipole using Tikhonov regularization," in Proc. OIPE Conf., Grenoble, France, 2004, p. 45.

[4] L.-L. Rouve et al., "Application of the spherical harmonic model to identify the magnetic state of a system and to extrapolate its signature," in Studies in Applied Electromagnetics and Mechanics 15, Electromagnetic Nondestructive Evaluation (III). Amsterdam, The Netherlands: IOS Press, 1999, pp. 136-144.

[5] J. P. Wikswo Jr. and K. R. Swinney, "Scalar multipole expansions and their dipole equivalents," J. Appl. Phys., vol. 56, pp. 3039-3049, 1984.

Manuscript received October 28, 2005 (e-mail: louis.schmerber@leg.ensieg.inpg.fr). 